\title{
Preoperative Needle Biopsy: Reasonable Targets, Benchmarking, and Quality Care in the Treatment of Breast Cancer
}

\author{
David J. Winchester, MD and David P. Winchester, MD \\ NorthShore University HealthSystem, Evanston Hospital, Evanston, IL
}

Breast cancer therapy has moved from more to less invasive methodologies, with greater emphasis on imageguided interventions that minimize toxicity and deformity. Defining the standard of care for breast cancer diagnosis and treatment is a means of elevating quality on a national level. This is a dynamic process, requiring constant evaluation of emerging technologies and clinical trial data. Dissemination and adoption of changing standards relate to many barriers, including access to care, geographic proximity to treatment facilities, reimbursement levels, and equipment expenses.

The era of a surgical evaluation consisting of an excisional biopsy or a needle-localized excision has become an historical footnote for most presentations of breast cancer. Many surgeons have also transitioned from palpation-guided needle biopsy to ultrasound-guided biopsy to increase the accuracy of their sampling procedure. Exceptions for the use of these approaches remain, but they have become rare and will continue to become less applicable. Initial core needle sampling is appropriately followed by surgical excision for discordant pathology, atypical ductal or lobular hyperplasia, lobular carcinoma-in-situ, incompletely ablated papillary lesions, and radial scars. Technical factors may limit the use of a needle biopsy, including inaccessible areas in proximity to the skin, chest wall, or prosthetic implant. Faint microcalcifications may not be amenable to stereotactic core needle biopsy. An initial open procedure is also appropriate for patients with nipple discharge in the absence of radiographic abnormalities, patients who require excision of benign-appearing lesions, and patients with suspicious lesions and comorbities that require

(C) Society of Surgical Oncology 2009

Published Online: 7 March 2009

D. J. Winchester, MD

e-mail: DWinchester2@northshore.org chronic anticoagulation or that diminish the impact of axillary staging.

For most breast cancer patients, Pocock and colleagues have enumerated much of the data showing favorable outcomes for needle diagnosis of breast cancer. ${ }^{1}$ These include reductions in operating room time, exposure to anesthesia, risk of infection, margin-positive excisions, lumpectomy extent and cosmetic deformity, and falsepositive sentinel node biopsy. Patient preference has been described as a reason for initial excision. However, a rational review of these endpoints should be sufficient to persuade most patients to proceed with a needle diagnosis.

Recognizing that a preoperative needle diagnosis offers many compelling advantages over an open procedure, it is a useful criterion for quality. In the report of Pocock et al., the retrospective retrieval of biopsy data for 396 patients took 10 hours, leading to an average of $1.5 \mathrm{~min}$ of time per patient. ${ }^{1}$ These data can be captured from a variety of sources, including medical reports and billing records. Thus, this criterion is both useful and readily accessible. Other proposed criteria for quality include the use of irradiation after breast-conserving surgery, adjuvant chemotherapy, adjuvant hormonal therapy, axillary evaluation in early-stage breast cancer, and conformity with the College of American Pathology reporting. ${ }^{2}$

As improvements in care are identified and the electronic medial record emerges, reimbursement has become easier to connect with quality endpoints. The National Cancer Data Base (NCDB) represents one data repository that collects information on $70 \%$ of the cancers treated in the United States. Treatment and pathology variables are entered into the NCDB, including use of radiation therapy, chemotherapy, hormone therapy, and axillary staging. With more sophisticated electronic data submission methods, inclusion of the diagnostic technique should be an attainable addition.

This endpoint should be measurable for hospitals, breast treatment centers, and individual practitioners. One 
potential mechanism for monitoring this endpoint at breast centers is through the National Accreditation Program of Breast Centers (NAPBC), a consortium of organizations involved in the care of breast cancer. The NAPBC has established 27 standards for evaluation and management, one of which is percutaneous needle biopsy. Compliance is verified by a site survey conducted every 3 years. By reviewing pathology reports, the surveyor can determine how the initial diagnosis was made and allow for justifiable exceptions. These standards also include the presence of a qualified or certified physician performing image-guided biopsies as determined by either the American College of Radiology or the American Society of Breast Surgeons. These criteria provide an opportunity to track, measure, and maintain quality on a large scale.

The usefulness of a preoperative needle biopsy does not appear to be related to stage or presentation, avoiding any interpretative inconsistencies related to geography or delay in diagnosis. On the basis of the report of Pocock et al. and those of others, a reasonable target for PND compliance would be $85 \%$, leaving room for appropriate exceptions. ${ }^{1,3}$ Measuring this benchmark (among others) will help the treating facility, physicians, and patients to recognize and maintain high-quality care in the treatment of breast cancer.

\section{REFERENCES}

1. Pocock B, Taback B, Klein L, et al. Preoperative needle biopsy as a quality measure in breast cancer surgery Ann Surg Oncol (in press).

2. National Quality Forum. Pre-voting review for "National Voluntary Consensus Standards for Diagnosis and Treatment of Breast and Colon Cancer." November 20, 2006. http://www.quality forum.org/pdf/cancer/txbreast-colonALLwebposting.pdf. Accessed February 6, 2009.

3. Tartter PI, Kaplan J, Bleiweiss I, et al. Lumpectomy margins, reexcision, and local recurrence of breast cancer Am J Surg 2000;179:81-5. 